\title{
The effects of psychiatric treatment on depression, anxiety, quality of life, and sexual dysfunction in patients with inflammatory bowel disease
}

This article was published in the following Dove Press journal:

Neuropsychiatric Disease and Treatment

24 March 2016

Number of times this article has been viewed

O Yanartas'
HT Kani'
E Bicakci
I Kilic
M Banzragch ${ }^{3}$
C Acikel
O Atug ${ }^{3}$
K Kuscu'
N Imeryuz
H Akin ${ }^{3}$
'Department of Psychiatry,
${ }^{2}$ Department of Internal
Medicine, ${ }^{3}$ Department of
Gastroenterology, ${ }^{4}$ Department of
Internal Medicine, Marmara University
School of Medicine, Istanbul,
'Department of Public Health,
Gulhane Military Medical Academy,
Ankara, Turkey

Correspondence: $O$ Yanartas Department of Psychiatry, Marmara University School of Medicine, Istanbul, Turkey

Tel +905446121230

Fax +90 2I 66570695

Email omeryanartas@yahoo.com
Objective: Depression and anxiety are common disorders in inflammatory bowel disease (IBD). Our aim is to prospectively determine the effect of psychiatric treatment on scores for depression, anxiety, quality of life (QoL), and sexual dysfunction in an outpatient population diagnosed with IBD and also anxiety and/or depression disorder.

Patients and methods: Patients who scored higher than the cutoff point on the Hospital Anxiety Depression Scale were referred for further structured psychiatric evaluation and determination of the need for psychiatric drug treatment. Patients who underwent drug therapy completed Short Form-36 (SF-36) and the Arizona Sexual Experience Scale at baseline and after 6 months of follow-up.

Results: Major depressive disorder and generalized anxiety disorder were the most common diagnoses. After 6 months, 47 patients had completely adhered to drug treatment (group A), whereas 20 were nonadherent (group B). In group A, all domains of SF-36, Arizona Sexual Experience Scale, depression/anxiety scores, and Crohn's disease activity index were statistically improved after treatment when compared with the baseline. In group B, the three domains of SF-36, platelet count, and mean corpuscular volume were worse between baseline and at 6 months.

Conclusion: In IBD patients having any psychiatric disorder, 6 months of antidepressant drug treatment is associated with an improvement in depression, anxiety, QoL, and sexual functioning scores, as well as an improvement in Crohn's disease activity index. On the other hand, insufficient psychiatric treatment seems to be related to a poor QoL.

Keywords: anxiety, depression, sexual dysfunction, psychiatric treatment, inflammatory bowel disease

\section{Introduction}

Inflammatory bowel diseases (IBDs), including Crohn's disease (CD) and ulcerative colitis (UC), are chronic, idiopathic inflammatory diseases of the gastrointestinal tract. IBD is related to increased morbidity and reduced quality of life (QoL). ${ }^{1,2}$ IBD is also linked to direct health care costs, imposing an economic burden of \$6 billion in the US, ${ }^{3}$ and indirect costs related to lost productivity and number of days absent from work.

Levels of depression and anxiety disorders in patients with IBD are reported to be higher than in those with colon cancer. ${ }^{4}$ In addition, they are linked with a poor QoL, decreased drug treatment adherence, more frequent recurrence of the disease, and more common sexual dysfunction. ${ }^{4-6}$ It is suggested that depression, anxiety, and impaired QoL may exert a negative influence on the course of IBD. ${ }^{4,5}$ On the other hand, aggressive IBD is claimed to be independently related to depression; ${ }^{7}$ so, there seems to be a bidirectional relationship between psychological issues and IBD. ${ }^{5,8,9}$ 
As one of the psychological models, insecure attachment is linked to deterioration of QoL with high anxiety and low selfesteem, not only diminishing interpersonal relationships but also impairing social interactions in the IBD patient group. ${ }^{2,8,9}$ Usage of antidepressants to treat concomitant depression in patients with IBD was found to be associated with a decrease in relapse rates, steroid use, and number of endoscopies. ${ }^{10}$ Most of the current literature studies address the need to determine the effects of antidepressant drug treatment on the IBD patient group in prospectively designed clinical studies. A recent literature review that was focused on antidepressants and IBD pointed to the presence of significant barriers to make a definitive statement on antidepressant drug efficacy for the mental and somatic status of patients with IBD due to the poor quality of available research. ${ }^{5}$ There are some cross-sectional studies in the IBD patient group measuring depression and/or anxiety as a symptom level. .,5,11,12 $^{2}$ However, most of these studies did not use the diagnostic criteria from the Diagnostic and Statistical Manual of Mental Disorders (DSM) or International Statistical Classification of Diseases and Related Health Problems to detect depression and/or anxiety disorder as a diagnosis in their study population. There is no prospective interventional study to evaluate the effects of antidepressant drug treatment after confirming the existence of a diagnosis of depressive and/or anxiety disorder in the IBD patient population that uses a structured psychiatric interview carried out by a psychiatrist according to DSM criteria.

The aim of this study was to assess the effects of 6 months of antidepressant drug treatment on scores of depression, anxiety, QoL, and sexual dysfunction in an IBD outpatient population having diagnosed with anxiety and/or depression disorder.

\section{Patients and methods}

\section{Participants}

This study was designed as a prospective one among patients followed up in the IBD-specific gastroenterology outpatient clinic at Marmara University Hospital between June 1, 2013, and June 1, 2014. Patients were included in the study consecutively during their admission to the outpatient clinic. Inclusion criteria were as follows: patients with IBD followed in our outpatient IBD clinics (with a diagnosis of CD or UC), willingness to participate in the study, and ability to sign an informed consent.

The IBD diagnosis had been established after endoscopic, histological, and radiographic investigations. ${ }^{13}$ Exclusion criteria were as follows: aged $<17$ years, illiteracy, physical handicaps (ie, blindness), ongoing psychiatric treatment, and mental retardation. Written informed consent was obtained from all the participants. The study was performed according to the principles of the Declaration of Helsinki and in accordance with the Medical Research Involving Human Subjects Act (WMO). The Marmara University School of Medicine Local Ethics Committee approved the study.

\section{Study design}

In the screening phase of the study, a sociodemographic form and the Hospital Anxiety and Depression Scale (HADS) were given to the patients with the aim of detecting anxiety and depression (Figure 1). Patients with scores above the cutoff point for depression and anxiety were referred within a week to a psychiatrist certified to carry out the Structured Clinical Interview for DSM-IV Axis I Disorders (SCID-I). After a psychiatric interview using SCID-I, patients completed Short Form-36 (SF-36) and Arizona Sexual Experience Scale (ASEX) tests for assessing QoL and sexual dysfunction. Individuals diagnosed with any psychiatric disorder were offered psychiatric treatment. Crohn's disease activity index (CDAI) and modified Mayo score (MMS) for the assessment of disease activity in patients with $\mathrm{CD}$ or UC, respectively, along with C-reactive protein (CRP), complete blood count, and routine blood biochemistry were collected on all visits. As well as these laboratory data, demographic data and data related to disease history (current and past drugs, operation history, disease localization and behavior of the IBD, presence of perianal disease and fistulas) were gathered for the assessment of inflammation in both patients with UC and patients with CD. All subjects were followed up for adverse effects of psychiatric drug therapy. All the psychiatric tests - HADS, SF-36, and ASEX - along with the CDAI and MMS IBD-related indices, and the laboratory data, including CRP and complete blood count, were performed at the beginning of treatment (baseline visit) and at 6 months (final visit) of the treatment period (Figure 1). Patients filled out the questionnaire in a separate room and were assisted by a gastroenterologist and a psychiatrist if they needed any help.

\section{Assessments}

\section{Sociodemographic form}

The sociodemographic form was completed by the researchers who put the related questions directly to the participants. It included age, income (classed subjectively as low, medium, or high), marital status, education (years), operational status, past psychiatric history, familial psychiatric treatment history, and sleep disturbance related to psychiatric treatment. 


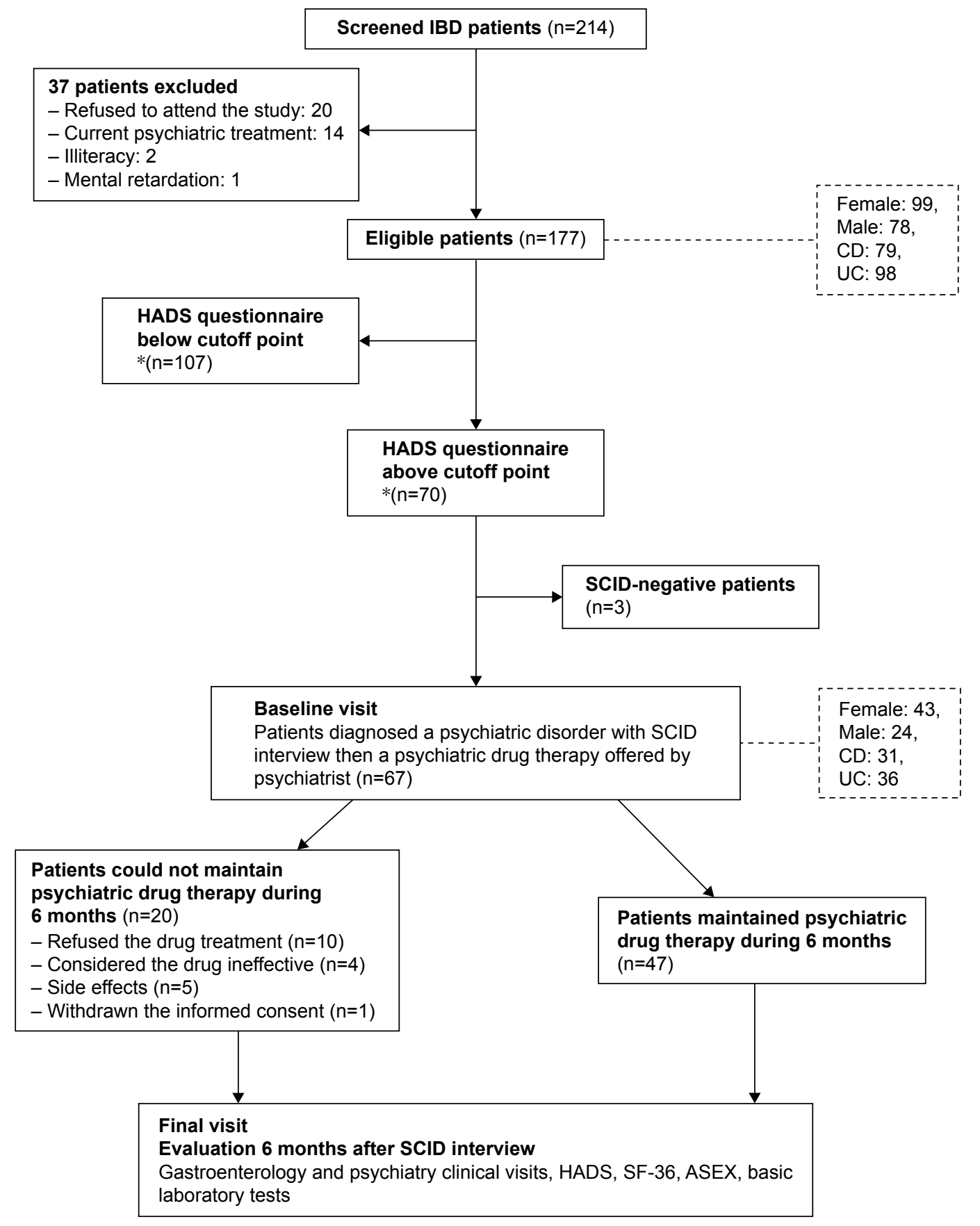

Figure I Flow chart of the study: the effects of psychiatric treatment in inflammatory bowel disease patients.

Notes: *HADS anxiety score $>7$ described as depression and anxiety score $>10$ described as anxiety.

Abbreviations: ASEX, Arizona Sexual Experience Scale; CD, Crohn's disease; DSM-IV, Diagnostic and Statistical Manual of Mental Disorders, fourth edition; HADS, Hospital Anxiety and Depression Scale; IBD, inflammatory bowel disease; SCID, Structured Clinical Interview for DSM-IV Axis I Disorders; SF-36, Short Form-36; UC, ulcerative colitis.

\section{Structured clinical interview for DSM-IV axis I disorders}

The SCID-I is a psychiatric diagnostic tool that is a semistructured interview technique for determining the presence of DSM-IV Axis I diagnoses. The SCID-I interview increases diagnostic accuracy significantly and can only be performed by a certified psychiatrist/psychologist/nurse.
The scale was developed in 1997 , and its validity and reliability have been established. ${ }^{14,15}$

\section{Hospital Anxiety and Depression Scale}

This is a self-reported test with multiple-choice questions that measure both the risk and the severity of anxiety and depression in patients with physical diseases. It has 
14 questions, ${ }^{16}$ the validity and reliability of this test have also been established. ${ }^{17}$ The cutoff points have been identified as above 10 for anxiety and above 7 for depression. ${ }^{17}$ Scores above the cutoff points predict the risk for depressive and/or anxiety disorders. ${ }^{16,17}$

\section{Quality of Life SF-36}

This is a validated self-reported scale commonly used to measure QoL and includes multiple-choice questions and a Likert scale. It was developed to evaluate QoL of patients with physical diseases. It is used to measure both negative and positive aspects of health status and is sensitive in detecting differences in disability. It evaluates eight categories of health with 36 items such as physical functioning, role limitations, social functioning, mental health, vitality, pain, and general health perception. ${ }^{18,19}$

\section{Arizona Sexual Experience Scale}

This is a self-reported questionnaire with five questions using Likert scales. It is designed to evaluate the major components of sexual dysfunction: drive, arousal, penile erection/vaginal lubrication, ability to reach orgasm, and satisfaction from orgasm..$^{20,21}$ The screening result from ASEX allows rapid detection of sexual dysfunction, and higher scores reflect impaired sexual dysfunction. A validity and reliability study has been performed. ${ }^{21}$

\section{Assessment of IBD}

The disease activity of CD was scored with the CDAI. Higher scores mean a worsening of underlying CD activity. ${ }^{22}$ The MMS is another scoring system that calculates the disease activity of UC. ${ }^{23}$ Its three scoring subcategories are daily stool frequency, rectal bleeding, and the physician's global assessment. The endoscopic appearance of the mucosa is excluded from the partial Mayo score, as opposed to the Mayo score. Higher scores mean a worsening of underlying UC disease activity. ${ }^{24}$

\section{Statistical analysis}

The software program SPSS Version 22.0 (IBM Corporation, Armonk, NY, USA) was used for the statistical analysis. The psychiatric questionnaires and laboratory results within groups from baseline to final visit were analyzed by using a paired-samples $t$-test. Continuous variables were compared between groups by using the Mann-Whitney $U$ or Student's $t$-tests. To evaluate correlation between variables, Pearson's or Spearman's correlation coefficients were calculated. For all statistical analyses, the level of significance was defined as $P<0.05$.

\section{Logistic regression}

We first performed univariate analysis, and then suitable candidate variables were selected. A backward logistic regression elimination method was used to refine the logistic regression model. Some possible models were evaluated to determine the best model of multivariate logistic regression.

\section{Results}

\section{Sociodemographic features}

In the first phase of the study, 214 sequential IBD outpatients were invited to participate in the psychiatric questionnaires. Out of these, 37 patients were excluded according to the exclusion criteria (Figure 1). Of the eligible patients who completed the questionnaire (HADS), 99 were female and 98 had UC; the mean age was $40.71 \pm 12.71$ years. Sociodemographic data and data related to the underlying IBD are given for the whole group of 177 patients and for groups A and $\mathrm{B}$ in Table 1.

The HADS scores of 70 patients were above the cutoff point. Sixty-seven (95.71\%) patients who were above the HADS cutoff points were diagnosed as having any psychiatric disorder, as defined by SCID; the most common psychiatric diagnosis was major depressive disorder (43.3\%) (Table 2). Forty-seven patients completed the psychiatric treatment offered (group A), and 20 patients could not complete that treatment (group B) for various reasons (Figure 1).

\section{Comparison of parameters between baseline and final visit within groups $A$ and $B$}

Psychiatric questionnaires (HADS, SF-36, and ASEX) and biologic parameters, including disease activity indexes (CDAI and MMS) and laboratory results, were recorded at baseline and at final visits at 6 months. HAD-Anxiety (HAD-A), HAD-Depression (HAD-D), all domains of SF-36, ASEX, and hemoglobin plus CDAI parameters were statistically improved at the final visit in group A. Two subitems of SF-36 (SF-3 and SF-8), platelets, and mean corpuscular volume $(\mathrm{MCV})$ parameters were significantly worse in group B (Table 3 ).

\section{Comparison of variation in each parameter between baseline and final visit (between groups $A$ and $B$ )}

The mean change (the difference in each variable between baseline and final visit) was computed for each variable. In group $\mathrm{A}, \mathrm{HAD}-\mathrm{A}, \mathrm{HAD}-\mathrm{D}$, all domains of SF-36, and 
Table I Sociodemographic data and disease properties of patients with IBD

\begin{tabular}{|c|c|c|c|}
\hline & All patients with IBD & Group A & Group B \\
\hline & $(n=\mid 77)$ & $(n=47)$ & $(n=20)$ \\
\hline Age (years), mean $\pm S D$ & $40.7| \pm| 2.7 \mid$ & $39.06 \pm 11.67$ & $41.65 \pm 13.90$ \\
\hline Female sex $\mathrm{n}(\%)$ & $99(55.9)$ & $36(76.6)$ & $7(35.0)$ \\
\hline UC, n (\%) & $98(55.4)$ & $24(51.1)$ & $12(60.0)$ \\
\hline$C D, n(\%)$ & $79(44.6)$ & $23(48.9)$ & $8(40.0)$ \\
\hline \multicolumn{4}{|l|}{ Montreal classification } \\
\hline \multicolumn{4}{|l|}{ Age at diagnosis of CD, $\mathrm{n}(\%)$} \\
\hline A2 I7 $<40$ years & $64(81.0)$ & $19(82.6)$ & $5(62.5)$ \\
\hline $\mathrm{A} 3 \geq 40$ years & $15(19.0)$ & $4(17.4)$ & $3(37.5)$ \\
\hline \multicolumn{4}{|l|}{ Localization of CD, n (\%) } \\
\hline LI ileal & $37(46.8)$ & $13(56.6)$ & $3(37.5)$ \\
\hline L2 colonic & $8(10.1)$ & I (4.3) & $0(0.0)$ \\
\hline L3 ileocolonic & $33(41.8)$ & $8(34.8)$ & $5(62.5)$ \\
\hline L4 isolated upper disease & $\mathrm{I}(\mathrm{I} .3)$ & I (4.3) & $0(0.0)$ \\
\hline \multicolumn{4}{|l|}{ Behavior of CD, $n(\%)$} \\
\hline BI nonstructuring, nonpenetrating & $47(59.5)$ & $16(69.6)$ & $5(62.5)$ \\
\hline B2 structuring & $9(11.4)$ & I (4.3) & I (I2.5) \\
\hline B3 penetrating & $23(29.1)$ & $6(26.1)$ & $2(25.0)$ \\
\hline P perianal disease modifier & $16(9.0)$ & $7(14.9)$ & $5(25.0)$ \\
\hline \multicolumn{4}{|l|}{ Extent of UC, $n(\%)$} \\
\hline EI ulcerative proctitis & $33(33.7)$ & $4(16.7)$ & $4(33.3)$ \\
\hline E2 left-sided UC (distal UC) & $33(33.7)$ & $9(37.5)$ & $4(33.3)$ \\
\hline E3 extensive UC (pancolitis) & $32(32.6)$ & II (45.8) & $4(33.3)$ \\
\hline Smoking & $39(22.0)$ & $10(21.2)$ & $8(40.0)$ \\
\hline Alcohol consumption & $9(5.0)$ & $2(4.2)$ & $3(15.0)$ \\
\hline \multicolumn{4}{|l|}{ Drug usage, $n(\%)$} \\
\hline Anti-TNF & $56(31.6)$ & $21(44.7)$ & $4(20.0)$ \\
\hline Corticosteroids & $14(7.9)$ & $3(6.4)$ & I $(5.0)$ \\
\hline Azathioprin & $80(45.2)$ & $22(46.8)$ & $7(35.0)$ \\
\hline Methotrexate & $4(2.3)$ & $7(14.9)$ & I (5.0) \\
\hline Mesalamine & $113(63.8)$ & $31(66.0)$ & $14(70.0)$ \\
\hline Budesonide & $4(2.3)$ & $I(2.1)$ & $2(10.0)$ \\
\hline \multicolumn{4}{|l|}{ IBD severity at baseline visit, ${ }^{a} \mathrm{n}(\%)$} \\
\hline Inactive & $105(59.3)$ & $26(55.3)$ & $8(40.0)$ \\
\hline Mildly active & $53(29.9)$ & $14(29.8)$ & $9(45.0)$ \\
\hline Moderately active & $19(10.8)$ & $7(14.9)$ & $3(15.0)$ \\
\hline Past psychiatric treatment history & $37(20.9)$ & $12(25.5)$ & $4(20.0)$ \\
\hline Family history of a psychiatric disease & $18(10.2)$ & $8(17.0)$ & $3(15.0)$ \\
\hline Sleep disturbance symptoms & $80(45.2)$ & $35(74.5)$ & $12(60.0)$ \\
\hline
\end{tabular}

Notes: ${ }^{a}$ Disease severity indexes: CDAI =0-150 (inactive), I50-300 (moderate), >300 (severe), modified Mayo =0-I (inactive), 2-5 (moderate), >5 (severe). Group A: patients maintained psychiatric drug therapy during 6 months; Group B: patients could not maintain psychiatric drug therapy of 6 months.

Abbreviations: CD, Crohn's disease; CDAl, Crohn's disease activity index; IBD, inflammatory bowel disease; TNF, tumor necrosis factor; UC, ulcerative colitis.

Table 2 Distribution of the psychiatric diagnosis SCID

\begin{tabular}{ll}
\hline Distribution of the psychiatric diagnoses & $\mathbf{n}(\%)$ \\
\hline Major depressive disorder & $29(43.3)$ \\
Generalized anxiety disorder & $10(15.0)$ \\
Adjustment disorder & $9(13.4)$ \\
Panic disorder & $5(7.5)$ \\
Dysthymic disorder & $4(6.0)$ \\
Generalized anxiety disorder + major depressive disorder & $5(7.5)$ \\
Minor depressive disorder & $2(2.9)$ \\
Bipolar disorder & $2(2.9)$ \\
Obsessive compulsive disorder & $1(1.5)$ \\
Total & $67(100.0)$ \\
\hline
\end{tabular}

Abbreviations: DSM-IV, Diagnostic and Statistical Manual of Mental Disorders, fourth edition; SCID, Structured Clinical Interview for DSM-IV Axis I Disorders.
ASEX variables were improved at the final visit compared to baseline visit. In group B, all domains of SF-36, ASEX 2, 3 , and 5, and ASEX total were worse at the final visit. If we compared the mean of the change in each variable between groups A and B, the variation of HAD-A, HAD-D, all SF-36 domains, and all ASEX parameters was statistically more improved in group A than in group B. The mean change in platelet numbers was statistically higher in group B compared to group A. For all the other biologic parameters (CDAI, MMS, CRP, white blood count, hemoglobin, hematocrit, and $\mathrm{MCV}$ ), the mean change between baseline and final visit was statistically insignificant (Table 4). 
Table 3 Comparison of HAD-A, HAD-D, SF-36, ASEX, and laboratory results of baseline (base) and final sixth month (final) visits in inflammatory bowel disease outpatient population who diagnosed a psychiatric disorder with SCID

\begin{tabular}{|c|c|c|c|c|c|}
\hline \multicolumn{3}{|l|}{ Group A } & \multicolumn{3}{|c|}{ Group B } \\
\hline$(n=47)$ & & & $(n=20)$ & & \\
\hline Object & Mean \pm SD & $P$-value & Object & Mean \pm SD & $P$-value \\
\hline HAD-A & & $<0.001$ & HAD-A & & 0.811 \\
\hline Base & $12.38 \pm 4.38$ & & Base & $11.40 \pm 4.60$ & \\
\hline Final & $5.97 \pm 4.45$ & & Final & $11.05 \pm 4.40$ & \\
\hline HAD-D & & $<0.001$ & HAD-D & & 0.203 \\
\hline Base & $|0.62 \pm 3.6|$ & & Base & $11.55 \pm 2.85$ & \\
\hline Final & $3.53 \pm 4.0 \mathrm{I}$ & & Final & $10.15 \pm 3.51$ & \\
\hline Sf-I & & $<0.001$ & Sf-I & & 0.098 \\
\hline Base & $51.44 \pm 26.49$ & & Base & $68.50 \pm 31.37$ & \\
\hline Final & $73.00 \pm 22.69$ & & Final & $54.00 \pm 27.22$ & \\
\hline Sf-2 & & $<\mathbf{0 . 0 0 1}$ & $\mathrm{Sf}-2$ & & 0.285 \\
\hline Base & $25.00 \pm 34.12$ & & Base & $46.25 \pm 43.13$ & \\
\hline Final & $60.55 \pm 34.74$ & & Final & $32.50 \pm 30.45$ & \\
\hline Sf-3 & & $<0.001$ & Sf-3 & & 0.050 \\
\hline Base & $42.7 I \pm 24.24$ & & Base & $58.70 \pm 22.49$ & \\
\hline Final & $58.11 \pm 21.26$ & & Final & $44.45 \pm 22.75$ & \\
\hline Sf-4 & & $<0.001$ & Sf-4 & & 0.052 \\
\hline Base & $28.17 \pm 16.64$ & & Base & $40.65 \pm|8.7|$ & \\
\hline Final & $48.88 \pm 22.30$ & & Final & $27.00 \pm 24.23$ & \\
\hline Sf-5 & & $<\mathbf{0 . 0 0 1}$ & Sf-5 & & 0.196 \\
\hline Base & $33.55 \pm 19.29$ & & Base & $43.75 \pm 22.87$ & \\
\hline Final & $57.66 \pm 23.75$ & & Final & $34.50 \pm 21.57$ & \\
\hline Sf-6 & & $<0.001$ & Sf-6 & & 0.478 \\
\hline Base & $42.50 \pm 23.89$ & & Base & $47.50 \pm 21.68$ & \\
\hline Final & $61.38 \pm 20.78$ & & Final & $41.25 \pm 24.70$ & \\
\hline Sf-7 & & $<0.001$ & Sf-7 & & 0.135 \\
\hline Base & $29.62 \pm 38.4 I$ & & Base & $43.33 \pm 37.61$ & \\
\hline Final & $65.17 \pm 38.24$ & & Final & $26.66 \pm 29.81$ & \\
\hline Sf-8 & & $<0.001$ & Sf-8 & & 0.042 \\
\hline Base & $40.44 \pm 16.60$ & & Base & $46.40 \pm 16.74$ & \\
\hline Final & $62.04 \pm 17.96$ & & Final & $32.40 \pm 19.33$ & \\
\hline ASEX I & & $<0.001$ & ASEX I & & 0.766 \\
\hline Base & $3.95 \pm 1.31$ & & Base & $3.08 \pm 1.38$ & \\
\hline Final & $2.48 \pm 1.27$ & & Final & $2.92 \pm 1.32$ & \\
\hline ASEX 2 & & $<0.00$ I & ASEX 2 & & 0.625 \\
\hline Base & $3.69 \pm 1.47$ & & Base & $2.91 \pm 1.22$ & \\
\hline Final & $2.38 \pm 1.47$ & & Final & $3.18 \pm 1.25$ & \\
\hline ASEX 3 & & $<\mathbf{0 . 0 0 1}$ & ASEX 3 & & 1.000 \\
\hline Base & $3.51 \pm 1.39$ & & Base & $2.77 \pm 1.16$ & \\
\hline Final & $2.33 \pm 1.28$ & & Final & $2.76 \pm 1.23$ & \\
\hline ASEX 4 & & $<0.001$ & ASEX 4 & & 0.820 \\
\hline Base & $3.55 \pm 1.5 \mid$ & & Base & $3.00 \pm 0.85$ & \\
\hline Final & $2.26 \pm 1.42$ & & Final & $2.91 \pm 1.31$ & \\
\hline ASEX 5 & & $<0.001$ & ASEX 5 & & 0.202 \\
\hline Base & $3.43 \pm 1.40$ & & Base & $2.33 \pm 1.15$ & \\
\hline Final & $2.05 \pm \mid .31$ & & Final & $3.08 \pm 1.31$ & \\
\hline ASEX T & & $<\mathbf{0 . 0 0 1}$ & ASEX T & & 0.210 \\
\hline Base & $17.89 \pm 6.01$ & & Base & $14.00 \pm 4.24$ & \\
\hline Final & $11.43 \pm 5.62$ & & Final & $16.54 \pm 4.78$ & \\
\hline CRP & & 0.324 & CRP & & 0.949 \\
\hline Base & $6.58 \pm 13.89$ & & Base & $4.30 \pm 3.79$ & \\
\hline Final & $4.6 I \pm 4.03$ & & Final & $4.35 \pm 3.47$ & \\
\hline
\end{tabular}


Table 3 (Continued)

\begin{tabular}{|c|c|c|c|c|c|}
\hline \multicolumn{3}{|c|}{ Group A } & \multicolumn{3}{|c|}{ Group B } \\
\hline \multicolumn{3}{|l|}{$(n=47)$} & \multicolumn{3}{|l|}{$(n=20)$} \\
\hline Object & Mean \pm SD & $P$-value & Object & Mean \pm SD & $P$-value \\
\hline$\overline{\mathrm{WBC}}$ & & $0.58 \mathrm{I}$ & WBC & & 0.576 \\
\hline Base & $6.50 \pm 1.94$ & & Base & $7,161 \pm 2,114$ & \\
\hline Final & $6.39 \pm 1.93$ & & Final & $7,483 \pm 2,136$ & \\
\hline $\mathrm{Hb}$ & & 0.023 & $\mathrm{Hb}$ & & 0.102 \\
\hline Base & $12.40 \pm 1.60$ & & Base & $13.49 \pm 1.59$ & \\
\hline Final & $12.86 \pm 1.15$ & & Final & $13.72 \pm 1.58$ & \\
\hline $\mathrm{Htc}$ & & 0.429 & $\mathrm{Htc}$ & & 0.477 \\
\hline Base & $37.79 \pm 4.30$ & & Base & $40.65 \pm 4.25$ & \\
\hline Final & $38.25 \pm 3.04$ & & Final & $40.96 \pm 4.32$ & \\
\hline Plt & & 0.112 & Plt & & $0.04 I$ \\
\hline Base & $290,097 \pm 92,872$ & & Base & $269,000 \pm 83,882$ & \\
\hline Final & $279,707 \pm 91,409$ & & Final & $293,444 \pm I 13,376$ & \\
\hline MCV & & 0.281 & $\mathrm{MCV}$ & & $0.04 I$ \\
\hline Base & $85.57 \pm 7.37$ & & Base & $87.72 \pm 4.40$ & \\
\hline Final & $84.69 \pm 7.69$ & & Final & $86.35 \pm 3.21$ & \\
\hline MMS & & 0.054 & MMS & & 0.464 \\
\hline Base & $2.7 I \pm 3.05$ & & Base & $2.78 \pm 3.42$ & \\
\hline Final & $0.94 \pm 1.91$ & & Final & $1.77 \pm 1.98$ & \\
\hline CDAI & & 0.011 & CDAl & & 0.710 \\
\hline Base & $197.41 \pm 130.60$ & & Base & $58.50 \pm 74.94$ & \\
\hline Final & $101.08 \pm 65.88$ & & Final & $83.50 \pm 62.68$ & \\
\hline
\end{tabular}

Notes: ASEX I, drive; ASEX 2, arousal; ASEX 3, penile erection/vaginal lubrication; ASEX 4, ability to reach orgasm; ASEX 5, satisfaction from orgasm; base, baseline visit; final, final visit; SfI: SF-36: physical functioning; Sf2, SF-36: role physical; Sf3, SF-36: bodily pain; Sf4, SF-36: general health; Sf5, SF-36: vitality; Sf6, SF-36: social functioning; Sf7, SF-36: role emotional; Sf8, SF-36: mental health. Group A: patients maintained psychiatric drug therapy during 6 months; Group B: patients could not maintain psychiatric drug therapy of 6 months. All boldface values are statistically significant values.

Abbreviations: ASEX T, ASEX total; CD, Crohn's disease; CDAl, Crohn's disease activity index; CRP, C reactive protein; DSM-IV, Diagnostic and Statistical Manual of Mental Disorders, fourth edition; HAD-A, Hospital Anxiety and Depression Scale Anxiety score; HAD-D, Hospital Anxiety and Depression Scale Depression score; Hb, hemoglobin; Htc, hematocrit; IBD, inflammatory bowel disease; MCV, mean corpuscular volume; MMS, modified Mayo score; PIt, platelet; SCID, Structured Clinical Interview for DSM-IV Axis I Disorders; SF-36, Short Form-36; UC, ulcerative colitis; WBC, white blood cell.

\section{Correlation of mean change in variables within group $A$ at 6 months}

The mean changes after 6 months (difference between final visit and baseline visit results) in HAD-D were negatively correlated with the mean changes of all the QoL items (SF-36) and positively correlated with the results of mean changes in all ASEX items.

The mean changes in HAD-A were positively correlated with the mean changes in all ASEX items, except for ASEX 2.

The mean changes in all ASEX items were negatively correlated with the mean changes in all SF-36 domains.

The mean changes in MMS were negatively correlated with QoL items (SF-2), hemoglobin, and hematocrit and positively correlated with platelet counts. The mean changes in CDAI were negatively correlated with the mean changes in QoL items (SF-2 and SF-3).

The mean changes in CRP were positively correlated with MMS and CDAI and negatively correlated with hemoglobin and hematocrit. The mean changes in hemoglobin and hematocrit were positively correlated with MCV and negatively correlated with platelet counts.

\section{Results of logistic regression models}

We performed a logistic regression model for the prediction of diagnosis of any psychiatric disorder in the outpatient IBD population. We ended up with two variables: that is, having sleep problems $(-2.218, P<0.001$, odds ratio [OR] [95\% confidence interval $\{\mathrm{CI}\}], 0.109[0.041-0.290])$ and having a past psychiatric family history $(-2.355, P: 0.009$, OR $[95 \%$ CI], 0.095 [0.016-0.548]).

We performed a second logistic regression model to determine the factors for predicting which patients would complete the psychiatric treatment offered. We ended up with two variables, the first being female sex $(1,632, P: 0.047$, OR [95\% CI], 5,115 [1.021-25.617]) and the second being the presence of comorbid health conditions (2,957, $P: 0.009$, OR [95\% CI], 19,240 [2.079-179.074]). 
Table 4 Comparison of each item's mean change between baseline and 6-month visits, between group $A$ and group $B$

\begin{tabular}{|c|c|c|c|}
\hline \multirow[t]{2}{*}{ Parameters } & \multirow{2}{*}{$\begin{array}{l}\text { Group A } \\
n=47 \\
(\text { mean } \pm \text { SD) }\end{array}$} & \multirow{2}{*}{$\begin{array}{l}\text { Group B } \\
\mathbf{n}=20 \\
\text { (mean } \pm \text { SD) }\end{array}$} & \multirow[t]{2}{*}{$P$-value } \\
\hline & & & \\
\hline SF-I & $21.5 \pm 31.8$ & $-14.5 \pm 37.2$ & $<\mathbf{0 . 0 0 1}$ \\
\hline SF-2 & $35.5 \pm 41.5$ & $-13.7 \pm 55.8$ & 0.001 \\
\hline SF-3 & $15.3 \pm 24.6$ & $-14.2 \pm 30.4$ & 0.001 \\
\hline SF-4 & $20.7 \pm 21.5$ & $-13.6 \pm 29.4$ & $<0.001$ \\
\hline SF-5 & $18.8 \pm 28.9$ & $-6.2 \pm 38.5$ & 0.010 \\
\hline SF-6 & $24.1 \pm 26.6$ & $-9.2 \pm 30.8$ & $<0.001$ \\
\hline SF-7 & $35.5 \pm 42.8$ & $-16.6 \pm 47.7$ & $<0.001$ \\
\hline SF-8 & $21.6 \pm 24.8$ & $-14.0 \pm 28.6$ & $<0.001$ \\
\hline HAD-A & $-6.4 \pm 6.6$ & $-0.35 \pm 6.4$ & 0.001 \\
\hline HAD-D & $-5.0 \pm 5.5$ & $-1.4 \pm 4.7$ & 0.017 \\
\hline ASEX I & $-1.4 \pm 1.6$ & $-0.15 \pm 1.8$ & 0.021 \\
\hline ASEX 2 & $-1.3 \pm 1.5$ & $0.27 \pm 1.79$ & 0.011 \\
\hline ASEX 3 & $-1.1 \pm 1.5$ & $0.00 \pm 1.4$ & 0.027 \\
\hline ASEX 4 & $-1.2 \pm 1.5$ & $-0.083 \pm 1.8$ & 0.021 \\
\hline ASEX 5 & $-1.3 \pm 1.5$ & $0.75 \pm 1.9$ & 0.002 \\
\hline ASEX T & $-6.4 \pm 5.8$ & $2.5 \pm 6.2$ & $<0.001$ \\
\hline MMS & $-1.6 \pm 3.4$ & $-1.7 \pm 4.4$ & 0.926 \\
\hline CDAI & $-62.9 \pm 99.5$ & $-39.3 \pm 139.5$ & 0.570 \\
\hline CRP & $1.9 \pm 13.5$ & $0.04 \pm 3.1$ & 0.656 \\
\hline WBC & $-0.11 \pm 1.2$ & $0.32 \pm 2.3$ & 0.548 \\
\hline $\mathrm{Hb}$ & $0.4 \pm 1.2$ & $0.23 \pm 0.57$ & 0.928 \\
\hline $\mathrm{Htc}$ & $0.4 \pm 3.7$ & $0.31 \pm 1.81$ & 0.723 \\
\hline $\mathrm{MCV}$ & $-0.8 \pm 4.5$ & $-1.37 \pm 2.25$ & 0.459 \\
\hline Plt & $-10.39 \pm 40.93$ & $24.44 \pm 46.8$ & 0.016 \\
\hline
\end{tabular}

Notes: SEX I, drive; ASEX 2, arousal; ASEX 3, penile erection/vaginal lubrication; ASEX 4, ability to reach orgasm; ASEX 5, satisfaction from orgasm; SfI, SF-36: physical functioning; Sf2, SF-36: role physical; Sf3, SF-36: bodily pain; Sf4, SF-36: general health; Sf5, SF-36: vitality; Sf6, SF-36: social functioning; Sf7, SF-36: role emotional; Sf8, SF-36: mental health. Group A: patients maintained psychiatric drug therapy during 6 months; Group B: patients could not maintain psychiatric drug therapy of 6 months. All boldface values are statistically significant values.

Abbreviations: A ASEX T, ASEX total; CDAI, Crohn's disease activity index; CRP, C reactive protein; HAD-A, Hospital Anxiety and Depression Scale Anxiety score; HAD-D, Hospital Anxiety and Depression Scale Depression score; Hb, hemoglobin; Htc, hematocrit; MMS, modified Mayo score; MCV, mean corpuscular volume; Plt, platelet; WBC, white blood cell.

\section{Drugs used for psychiatric treatment}

The distribution of prescribed antidepressant agents was as follows: sertraline $21.0 \%$, escitalopram $15.8 \%$, bupropion $12.3 \%$, mirtazapine $12.3 \%$, paroxetine $10.6 \%$, venlafaxine $5.2 \%$, duloxetine $3.5 \%$, fluvoxamine $1.8 \%$, antidepressant combination treatment ([sertraline + mirtazapine] $7.0 \%$, [escitalopram + trazodone] 5.2\%, [venlafaxine + mirtazapine] $1.8 \%$ ), and second-generation antipsychotics plus antidepressants (sertraline + quetiapine) 3.5\%.

\section{Side effects}

Sixty-seven patients were offered antidepressant treatment. The frequency of side effects related to antidepressant usage was nearly one-third ( $\mathrm{n}=19,33.3 \%$ ) of all patients. However, of the 19 patients who had any type of drug-related side effect, only five (26.3\%) discontinued the antidepressant medication. The causes of discontinuation of antidepressant therapy were new-onset of sexual dysfunction in three cases, weight gain in one case, and fatigue and drowsiness in another case. The most common side effect was drowsiness and fatigue (five cases); the others were post-antidepressant therapy sexual dysfunction (four cases), weight gain (four cases), insomnia (three cases), anxiety (two cases), and nausea (one case).

\section{Discussion}

The need for psychiatric treatment was previously demonstrated to be more common in patients with IBD than in the general population. In a previous study from Canada, it is documented that only $40 \%$ of IBD patients with diagnoses of depression disorder were treated with antidepressant agents. ${ }^{25}$ It is stated that patients with IBD were not given sufficient levels of appropriate psychiatric treatment. ${ }^{25}$ In the screening phase of our study, we found that 14 patients were receiving some form of current psychiatric treatment. Strikingly, we detected at least 67 additional patients who needed psychiatric treatment but were undertreated (82.8\%). Both gastroenterologists and other IBD practitioners, including IBD surgeons, should keep in mind the need for screening for psychiatric symptoms. ${ }^{5,26}$

Almost all the patients with IBD (67 of 70) who had a HADS questionnaire score above the cutoff level had a comorbid psychiatric disorder, as confirmed by the following SCID-I interview. The SCID-I interviews were done by a certified psychiatrist, which increases the diagnostic accuracy of this structured interview. ${ }^{14,15}$ The proportion of psychiatric disorders (67 of 177 patients, 39.5\%) was found to be similar to that in other studies of an IBD population in remission. ${ }^{4,5,27}$ According to our study, in the outpatient IBD population, HADS was demonstrated to be a cheap, quick, and easily applied selfreporting paper-based tool that had a very high consistency with the results of SCID-I. For the provision of better health care for these patients, we suggest clinicians consider using HADS in their daily clinical practice.

In this study, the distribution of psychiatric diagnoses (Table 2) was in line with the previous literature findings. ${ }^{28-30}$ We suggest a routine psychiatrist consultation for patients at risk of a psychiatric disorder in order to reach an accurate diagnosis and to guide appropriate treatment.

In our study, the comparison of baseline and final visits within group A demonstrated that patients' adherence to adequate antidepressant treatment of 6 months duration significantly related to improvements in depression, anxiety, QoL, sexual dysfunction, and the biologic parameters 
of hemoglobin and CDAI. In group B, some parameters statistically worsened: that is, SF-3 and 8 and platelet count at the final visit (Table 3). In some nonprospective studies, psychological distress related to depression and anxiety has been associated with nonadherence to medical treatment and resulted in some detrimental effects on QoL, disease course, and treatment outcomes in the IBD patient population. ${ }^{11,27-29}$ Moreover, a predominantly insecure attachment style, characterized by attachment anxiety and attachment avoidance, was associated with decreased QoL in patients with IBD. ${ }^{2}$ Thus, psychological factors and interpersonal relationships may be important issues for the course of IBD.

In our study, we examined the outpatient IBD population. As expected, most of these patients were in remission, but some of them had varying degrees of underlying IBD activity. This reflected day-to-day real-life conditions in IBD outpatient clinics. In this setup, we additionally demonstrated that adherence to psychiatric treatment seems to increase QoL and improve sexual function in IBD outpatients. Antidepressants are reported to be effective drugs for treating depression and anxiety disorders in patients with IBD. ${ }^{4,31}$ There are studies that have shown their additional beneficial effects on symptoms such as pain and gut irritability. ${ }^{4,5}$ If patients with IBD have a comorbid mood disorder, antidepressant drug treatment is also claimed to improve the course of disease with a decrease in relapse rates and use of steroids in the first year of treatment. ${ }^{10}$

Here we revealed statistically significant improvements in some biologic parameters such as hemoglobin and CDAI after 6 months of psychiatric treatment. However, the clinical significance of this finding needs further clarification because most of our study groups were outpatients whose IBD was in remission. On the other hand, in group B, the patients not adhering to psychiatric drug therapy, platelet counts increased significantly. Although we did not measure adherence, one logical explanation for these findings may be increased adherence to the underlying IBD treatment due to these patients' decreased anxiety and depression scores. DiMatteo et $\mathrm{al}^{32}$ have demonstrated depression to be a risk factor for noncompliance with medical treatment. Another possible explanation for the improvement of biologic parameters with 6 months of psychiatric treatment may be the claimed anti-inflammatory effects of antidepressant drugs. ${ }^{4,5}$ Our study population includes mostly patients with IBD in remission, so it should have been more difficult to demonstrate any possible biologic benefit since most of the patients already have a near-normal daily status. On the other hand, these findings may be a harbinger of a possible stronger biologic benefit. In terms of evidence-based medicine, the specific impact of antidepressant agents on long-term IBD activity and biologic parameters is still unknown and debatable. ${ }^{33}$ Future prospective double-blind studies with a longer follow-up period are needed to address this issue.

It is well known that IBD and mental health problems may have a negative impact on both the QoL and sexual functioning of affected individuals., ${ }^{4,53}$ Timmer et $\mathrm{al}^{34}$ found that depressive mood was the strongest predictor of low sexual function (all domains of sexuality), and anxiety was associated with specific aspects of sexuality such as frequency of intercourse in female patients. We demonstrated that, between final and baseline visits, the variation in ASEX scores was correlated with the mean change in depression and anxiety scores and in QoL scores. However, we could not demonstrate any correlation between mean change in ASEX scores and mean change in biologic parameters. These results together lead us to suggest that improvement in all ASEX items is related to psychological parameters rather than biologic parameters.

Of note, most of the group A patients were female, and the decrease in depression scores seems to be associated with an improvement in sexual dysfunction scores, especially in the women's patient group. So our results were similar to those of Timmer et $\mathrm{al}^{34}$ in terms of female sex being associated with higher depression rate, higher sexual dysfunction rate, and greater adherence to psychiatric drug treatment.

An insecure attachment style characterized by attachment anxiety and avoidance may be one way to explain impaired interpersonal relationships in patients with IBD. In a recent study, impaired close interpersonal relationships related to both anxious and avoidant attachment styles are found to be important $t^{2,8,9}$ yet differential predictors of sexual function in men and women.

We computed the mean change in each variable between baseline and final visits within each group and then compared groups A and B. The means of all psychiatric test items were significantly improved at the final visits in group A compared to those of group B. These results demonstrate the importance of a sufficient psychiatric treatment period in the IBD patient population.

\section{Logistic regression analysis}

In previous studies on the IBD patient population, female sex, unmarried status, higher education level, and poor psychological well-being were factors predicting a psychiatric diagnosis. ${ }^{25,29}$ However, we could not demonstrate these factors for depression and/or anxiety disorder. In our patients, 
self-reported sleeping problems and past psychiatric family history were found to be related to a psychiatric disorder, compatible with the literature. ${ }^{5}$ Thus, this may be another important issue in patients with IBD. In our study, female sex and the presence of comorbid diseases were found to be related to prediction of adherence to psychiatric treatment. Treatment adherence is reported to be higher in female patients, ${ }^{35}$ and this may warn us about low psychiatric treatment adherence in male patients.

\section{Side effects}

Although $33.3 \%$ of the patients complained of at least one side effect with their antidepressant drug, only a small percentage of them $(8.7 \%)$ discontinued treatment because of such side effects. Treatment with antidepressant drugs seems to be effective and well tolerated for depressive and anxiety disorders in IBD outpatient population. Our prospective side-effect findings are in accordance with previous reports in cross-sectional and/or retrospective studies. ${ }^{5,33}$

\section{Limitations}

Our study has several limitations. First, we could not compare our findings according to the subtype of IBD because of the relatively small number of patients in group A and group B. Second, our follow-up period was relatively short. Another limitation might be the profile of patients related to IBD activity. Our study included patients with IBD from outpatient clinics, of whom most were in a state of remission. Although this profile is a good reflection of the real day-to-day life of an IBD outpatient clinic, even more individuals with flare-ups of IBD may need greater psychiatric attention.

\section{Conclusion}

To the best of our knowledge, this is the first prospective interventional follow-up study of IBD outpatients in whom psychiatric diagnoses were made by a psychiatrist using a structured clinical interview according to DSM-IV Text Revision (TR) to determine the effect of psychiatric treatment on scores for depression, anxiety, QoL, and sexual dysfunction. For the first time in literature, this study demonstrated that 6 months of psychiatric treatment in IBD outpatients with diagnoses of psychiatric depression and/or anxiety disorder are associated with improved sexual function. Statistically significant improvements of the scores for depression, anxiety, and QoL were also shown with antidepressant drug treatment in the same patient population. Psychiatric drug treatment was found to be associated with an improvement in CDAI in patients with IBD. These improvements were not observed in patients who could not adhere to the psychiatric treatment despite their need. Antidepressant agents seem to be well tolerated with minimal side effects. Depression and anxiety are common among IBD outpatients, and HADS seems be a cheap, helpful, easy-to-use instrument to screen for anxiety and depression in these patients. A multidisciplinary approach addressing depression and anxiety not only may improve patients' psychiatric symptoms but also may have some additional benefits on their QoL, sexual functioning, and disease activity related to IBD.

\section{Acknowledgments}

The authors wish to thank Yasin Bez, Zeynep Senkal Department of Psychiatry, Medical School of Marmara University, Istanbul, and Ayse Sakalli Kani, Department of Psychiatry, Medical School of Cerrahpasa, Istanbul University, Istanbul, for proofreading this manuscript.

\section{Disclosure}

The authors report no conflicts of interest in this work.

\section{References}

1. Cohen RD. The quality of life in patients with Crohn's disease. Aliment Pharmacol Ther. 2002;16(9):1603-1609.

2. Agostini A, Moretti M, Calabrese C, et al. Attachment and quality of life in patients with inflammatory bowel disease. Int J Colorectal Dis. 2014;29(10):1291-1296.

3. Kappelman MD, Rifas-Shiman SL, Porter CQ, et al. Direct health care costs of Crohn's disease and ulcerative colitis in US children and adults. Gastroenterology. 2008;135(6):1907-1913.

4. Graff LA, Walker JR, Bernstein CN. Depression and anxiety in inflammatory bowel disease: a review of comorbidity and management. Inflamm Bowel Dis. 2009;15(7):1105-1118.

5. Filipovic BR, Filipovic BF. Psychiatric comorbidity in the treatment of patients with inflammatory bowel disease. World J Gastroenterol. 2014; 20(13):3552-3563.

6. Marín L, Mañosa M, Garcia-Planella E, et al. Sexual function and patients' perceptions in inflammatory bowel disease: a case-control survey. J Gastroenterol. 2013;48(6):713-720.

7. Panara AJ, Yarur AJ, Rieders B, et al. The incidence and risk factors for developing depression after being diagnosed with inflammatory bowel disease: a cohort study. Aliment Pharmacol Ther. 2014;39(8): 802-810.

8. Agostini A, Rizzello F, Ravegnani G, et al. Adult attachment and early parental experiences in patients with Crohn's disease. Psychosomatics. 2010;51(3):208-215.

9. Maunder RG, Lancee WJ, Hunter JJ, Greenberg GR, Steinhart AH. Attachment insecurity moderates the relationship between disease activity and depressive symptoms in ulcerative colitis. Inflamm Bowel Dis. 2005; 11(10):919-926.

10. Goodhand JR, Greig FI, Koodun Y, et al. Do antidepressants influence the disease course in inflammatory bowel disease? A retrospective case-matched observational study. Inflamm Bowel Dis. 2012;18(7): 1232-1239.

11. Yanartas O, Bicakci E, Senkal Z, et al. Assessment of psychiatric symptoms and quality of life in patients with inflammatory bowel disease. JMOOD. 2014;4(3):115-121. 
12. Hauser W, Janke KH, Klump B, Hinz A. Anxiety and depression in patients with inflammatory bowel disease: comparisons with chronic liver disease patients and the general population. Inflamm Bowel Dis. 2011; 17:621-632.

13. Nikolaus S, Schreiber S. Diagnostics of inflammatory bowel disease. Gastroenterology. 2007;133(5):1670-1689.

14. First MB, Spitzer RL, Gibbon M, Williams JBW. User's Guide for the Structured Clinical Interview for DSM-IV Axis I Disorders SCID-I: Clinician Version. New York: American Psychiatric Press; 1997.

15. Özkürkçügil A, Aydemir Ö, Yıldız M, Esen Danacı A, Köroğlu E. DSM-IV eksen I bozuklukları için yapılandırılmış klinik görüşmenin Türkçe'ye uyarlanması ve güvenilirlik çalışması. [Structured clinical interview for DSM-IV axis I disorders-clinical version (SCID-CV) in Turkish: study of reliability]. İlaç ve Tedavi Dergisi. 1999;12(3): 233-236. Turkish.

16. Zigmond AS, Snaith RP. The hospital anxiety and depression scale. Acta Psychiatr Scand. 1983;67(6):361-370.

17. Aydemir Ö, Güvenir T, Küey L, Kültür S. [Vailidity and reliability of Turkish version of Hospital Anxiety and Depression Scale]. Hastane anksiyete ve depresyon ölçeği Türkçe formunun geçerlilik ve güvenilirliği. Türk Psikiyatri Dergisi. 1997;8(4):280-287. Turkish.

18. Ware JE Jr, Sherbourne CD. The MOS 36-item short-form health survey (SF-36). I. Conceptual framework and item selection. Med Care. 1992 30(6):473-483.

19. Koçyiğit H, Aydemir Ö, Fişek G, Ölmez N, Memiş A. [Validity and reliability of Turkish version of short form 36 (SF-36)]. Kisa Form 36 (KF-36)'nın Türkçe versiyonunun güvenilirliği ve geçerliliği. Ilaç ve tedavi dergisi. 1999;12(2):102-106. Turkish.

20. McGahuey CA, Gelenberg AJ, Laukes CA, et al. The Arizona Sexual Experience Scale (ASEX): reliability and validity. J Sex Marital Ther. 2000;26(1):25-40.

21. Soykan A. The reliability and validity of Arizona sexual experiences scale in Turkish ESRD patients undergoing hemodialysis. Int J Impot Res. 2004;16(1):531-534.

22. Best WR, Becktel JM, Singleton JW. Rederived values of the eight coefficients of the Crohn's Disease Activity Index (CDAI). Gastroenterology. 1979;77(4):843-846.

23. Schroeder KW, Tremaine WJ, Ilstrup DM. Coated oral 5-aminosalicylic acid therapy for mildly to moderately active ulcerative colitis A randomized study. $N$ Engl J Med. 1987;317(26):1625-1629.

24. Lewis JD, Chuai S, Nessel L, Lichtenstein GR, Aberra FN, Ellenberg JH. Use of the noninvasive components of the Mayo score to assess clinical response in ulcerative colitis. Inflamm Bowel Dis. 2008;14(12): $1660-1666$.
25. Fuller-Thomson E, Sulman J. Depression and inflammatory bowel disease: findings from two nationally representative Canadian surveys. Inflamm Bowel Dis. 2006;12(8):697-707.

26. Walker EA, Gelfand MD, Gelfand AN, Creed F, Katon WJ. The relationship of current psychiatric disorder to functional disability and distress in patients with inflammatory bowel disease. Gen Hosp Psychiatry. 1996;18(4):220-229.

27. Mittermaier C, Dejaco C, Waldhoer T, et al. Impact of depressive mood on relapse in patients with inflammatory bowel disease: a prospective 18-month follow-up study. Psychosom Med. 2004;66(1):79-84.

28. Nigro G, Angelini G, Grosso SB, Caula G, Sategna-Guidetti C. Psychiatric predictors of noncompliance in inflammatory bowel disease: psychiatry and compliance. J Clin Gastroenterol. 2001;32(1):66-68.

29. Walker JR, Ediger JP, Graff LA, et al. The Manitoba IBD cohort study: a population-based study of the prevalence of lifetime and 12-month anxiety and mood disorders. Am J Gastroenterol. 2008;103(8): 1989-1997.

30. Vidal A, Gómez-Gil E, Sans M, et al. The impact of anxiety and depression on relapse in patients with inflammatory bowel disease. Med Clin (Barc). 2009;132(8):298-302.

31. Mikocka-Walus AA, Gordon AL, Stewart BJ, Andrews JM. The role of antidepressants in the management of inflammatory bowel disease (IBD): a short report on a clinical case- note audit. J Psychosom Res. 2012;72(2):165-167.

32. DiMatteo MR, Lepper HS, Croghan TW. Depression is a risk factor for noncompliance with medical treatment: meta-analysis of the effects of anxiety and depression on patient adherence. Arch Intern Med. 2000; 24(14):2101-2107.

33. Mikocka-Walus A, Andrews JM. Attitudes towards antidepressants among people living with inflammatory bowel disease: an online Australia-wide survey. J Crohns Colitis. 2014;8(4):296-303.

34. Timmer A, Kemptner D, Bauer A, Takses A, Ott C, Fürst A. Determinants of female sexual function in inflammatory bowel disease: a survey based cross-sectional analysis. BMC Gastroenterol. 2008; $3(8): 1-10$

35. Woolley SB, Fredman L, Goethe JW, Lincoln AK, Heeren T. Hospital patients' perceptions during treatment and early discontinuation of serotonin selective reuptake inhibitor antidepressants. J Clin Psychopharmacol. 2010;30(6):716-719.
Neuropsychiatric Disease and Treatment

\section{Publish your work in this journal}

Neuropsychiatric Disease and Treatment is an international, peerreviewed journal of clinical therapeutics and pharmacology focusing on concise rapid reporting of clinical or pre-clinical studies on a range of neuropsychiatric and neurological disorders. This journa is indexed on PubMed Central, the 'PsycINFO' database and CAS,

\section{Dovepress}

and is the official journal of The International Neuropsychiatric Association (INA). The manuscript management system is completely online and includes a very quick and fair peer-review system, which is all easy to use. Visit http://www.dovepress.com/testimonials.php to read real quotes from published authors. 